\title{
Positronium production in cryogenic environments
}

\author{
B. S. Cooper, ${ }^{1}$ A. M. Alonso, ${ }^{1}$ A. Deller, ${ }^{1}$ L. Liszkay, ${ }^{2}$ and D. B. Cassidy ${ }^{1}$ \\ ${ }^{1}$ Department of Physics and Astronomy, University College London, Gower Street, London WC1E 6BT, United Kingdom \\ ${ }^{2}$ IRFU, CEA, University Paris-Saclay F-91191 Gif-sur-Yvette Cedex, France
}

(Received 22 January 2016; published 14 March 2016)

\begin{abstract}
We report measurements of positronium (Ps) formation following positron irradiation of mesoporous $\mathrm{SiO}_{2}$ films and $\mathrm{Ge}(100)$ single crystals at temperatures ranging from $12-700 \mathrm{~K}$. As both of these materials generate Ps atoms via nonthermal processes, they are able to function as positron-positronium converters at cryogenic temperatures. Our data show that such Ps formation is possibly provided the targets are not compromised by adsorption of residual gas. In the case of $\mathrm{SiO}_{2}$ films, we observe a strong reduction in the Ps formation efficiency following irradiation with UV laser light $(\lambda=243.01 \mathrm{~nm})$ below $250 \mathrm{~K}$, in accordance with previous observations of radiation-induced surface paramagnetic centers. Conversely, Ps emission from Ge is enhanced by irradiation with visible laser light $(\lambda=532 \mathrm{~nm})$ via a photoemission process that persists at cryogenic temperatures. Both mesoporous $\mathrm{SiO}_{2}$ films and Ge crystals were found to produce Ps efficiently in cryogenic environments. Accordingly, these materials are likely to prove useful in several areas of research, including Ps mediated antihydrogen formation conducted in the cold bore of a superconducting magnet, the production of Rydberg Ps for experiments in which the effects of black-body radiation must be minimized, and the utilization of mesoporous structures that have been modified to produce cold Ps atoms.
\end{abstract}

DOI: 10.1103/PhysRevB.93.125305

\section{INTRODUCTION}

Positronium (Ps) atoms can be produced in vacuum when positrons are implanted into various materials [1-6], known in this context as Ps converters. This can occur through several distinct mechanisms, some of which are nonthermal in nature. It is therefore expected that, in the absence of inhibiting processes, some Ps converters should function efficiently in cryogenic environments. Two good candidates for this are mesoporous films [7-9] and single-crystal semiconductors [10].

The generation of Ps in low-temperature environments is required for several different experimental endeavors. Examples include (1) the production of antihydrogen atoms by Ps impact with trapped antiprotons [11,12], (2) the formation of high- $n$ Rydberg states of Ps for gravity measurements $[13,14]$ or precision spectroscopy [15,16], and (3) the emission of low-energy Ps atoms into vacuum from engineered porous structures or semiconductors $[17,18]$. It is envisaged that antihydrogen formation would occur in or near the cold bore of a superconducting magnet. This may be achieved using a Cs-beam-based charge-exchange technique [19], but for some schemes direct Ps production from a solid-state target is required [20]. In order to conduct free-fall measurements or high-resolution spectroscopy, very long-lived Ps atoms may be required. This can be achieved by exciting to states with high principal quantum numbers $(\gtrsim 30)$, that may be sensitive to black-body radiation at room temperature [21]. Finally, modified converters that can increase Ps cooling rates, reduce Ps formation energies, or both, would have to be cooled accordingly.

An intriguing possibility offered by low-temperature, structured, mesoporous materials is a route to the formation of a Ps Bose-Einstein condensate (BEC), which has been discussed in the literature for some time [22,23]. A Ps BEC would serve as an ideal source of coherent Ps for matter-wave interferometry [24] and spectroscopy measurements, and is a prerequisite for the observation of stimulated annihilation, and perhaps even the production of a $\gamma$-ray laser [25-29]. Creating a Ps BEC requires cooling a dense ensemble of spin-polarized Ps atoms [30] below the BEC transition temperature. In the absence of an electrostatic or magneto-optical Ps trap, this must be done in a cavity in a solid-state material [23,31]. The highest Ps densities achieved to date [32] are of the order of $10^{16} \mathrm{~cm}^{-3}$, obtained in a mesoporous silica film. Increasing this density by two orders of magnitude would result in the relatively high [33] BEC transition temperature of $15 \mathrm{~K}$.

It may be possible to increase cooling rates for confined Ps using engineered porous structures that are designed to facilitate both cooling and collection of Ps atoms into a central void $[34,35]$. However, collisional cooling rates must necessarily decrease as the atoms approach thermalization [36,37], and a hybrid scheme utilizing both collisional and laser cooling may be necessary to reach the lowest temperatures allowed by the cavity [38]. In order to explore this possibility, it would be useful to understand how such materials function at low temperatures, and when irradiated with laser radiation. The latter point is particularly important since intense laser pulses may be required to laser cool a dense Ps gas in a cavity, owing to line-narrowing effects [39]. If a Ps gas could be laser cooled to the recoil limit [40] $(\sim 100 \mathrm{mK}$, which is higher than the $\sim 1 \mathrm{mK}$ Doppler limit for Ps because of the low mass) then the BEC transition would occur at a density close to what has already been achieved (i.e., around $10^{16} \mathrm{~cm}^{-3}$ ).

In this work, we study Ps formation from two different materials, mesoporous silica films, and Ge single crystals. Mesoporous $\mathrm{SiO}_{2}$ films have been shown to produce Ps with an energy and efficiency that does not depend on the sample temperature [41]. The energy of Ps emitted from Ge and $\mathrm{Si}$ semiconductors is also independent of the sample temperature [10]. Both $\mathrm{Si}$ and $\mathrm{Ge}$ crystals produce Ps via an excitonlike surface state $[10,18]$ in which the requisite electronic surface states are thermally activated. Thus the 
Ps formation efficiency from these materials does depend on the sample temperature. However, these electron states can also be populated optically [42], and we show here that laser irradiation at cryogenic temperatures results in efficient Ps formation from $\mathrm{Ge}$, with the added bonus that the photoemisison laser is also able to remove material adsorbed on the crystal surface.

\section{EXPERIMENTAL METHODS}

\section{A. Positron beam and $\gamma$-ray detection}

The apparatus and methods used in the present experiments have been described in detail elsewhere [43]. Positrons emitted from a ${ }^{22} \mathrm{Na}$ source are moderated with solid neon [44] and captured in a two-stage [45] Surko-type positron trap [46,47]. The trap is operated at a repetition rate of $1 \mathrm{~Hz}$, and the output consists of $\sim 10^{5}$ positrons/pulse. The positron pulse width is between $3-5 \mathrm{~mm}$ (FWHM), and is compressed in time to $\sim 6$ ns (FWHM) using a parabolic potential buncher [48]. These characteristics depend on the magnetic field strength in the target region and the distance between the bunching electrode and the target. Ps can be produced in vacuum by implanting positrons into suitable materials. The Ps atoms may then be probed with pulsed lasers, facilitating measurement of the velocity distribution via Doppler [49] or time-of-flight spectroscopy [50].

The amount of long-lived Ps formed by positron irradiation of $\mathrm{SiO}_{2}$ and $\mathrm{Ge}$ targets was measured using single-shot positron annihilation lifetime spectroscopy (SSPALS) [51]. This technique uses a fast $\gamma$-ray detector [52] coupled to an oscilloscope to measure annihilation radiation as a function of time, from which lifetime spectra are generated. A delayed $\gamma$-ray fraction $\left(f_{d}\right)$ is defined as the ratio of the integrated areas $B-C$ and $A-C$ of recorded lifetime spectra, where the values for $A, B$, and $C$ are $-6,35$, and 450 ns respectively (see Fig. 1).

If $B$ is positioned after the initial "prompt" peak arising from fast annihilation events, then the delayed fraction is less than the fraction of incident positrons that form ortho-

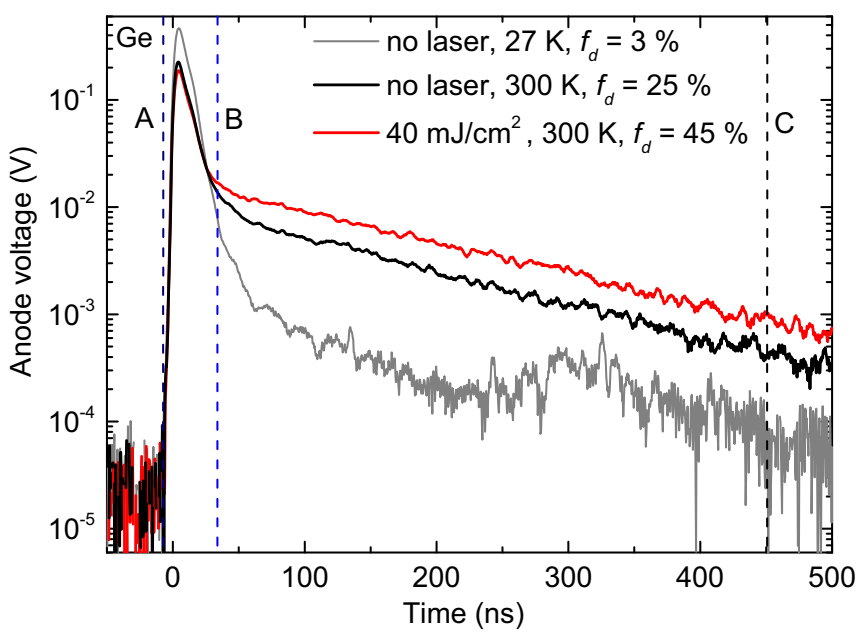

FIG. 1. Single-shot lifetime spectra measured with a Ge target, with and without a 532-nm photoemission laser, at 300 and $27 \mathrm{~K}$. The dashed vertical lines represent the time windows $A, B$, and $C$. Each spectrum is the average of 100 individual shots. positronium (o-Ps) $\left(f_{\mathrm{Ps}}\right)$. In general, $f_{\mathrm{Ps}}$ is at most $75 \%$, since spin statistics dictate that singlet and triplet Ps formation occurs in a 1:3 ratio [6]. An approximate estimate for the correction factor between $f_{d}$ and $f_{\mathrm{Ps}}$ is $f_{\mathrm{Ps}}=f_{d} \times \exp (B / 142)$. Thus $f_{\mathrm{Ps}} \sim 1.3 f_{d}$ for $B=35 \mathrm{~ns}$. The relationship between $f_{\mathrm{Ps}}$ and $f_{d}$ is considered in more detail elsewhere [10]. Examples of single-shot lifetime spectra recorded for various experimental conditions are shown in Fig. 1.

Changes in the lifetime spectra induced by a laser are quantified by the parameter $S_{\gamma}=\left(f_{d \text { (off) }}-f_{d \text { (on) })}\right) / f_{d \text { (off) }}$, where (on) and (off) refer to the laser. The (off) case may be defined as the laser being present but off resonance, or not present at all. For the data shown in Fig. 1, $f_{d}$ for the spectra with the least $o$-Ps (no laser, $27 \mathrm{~K}$ ) is $3 \pm 1 \%$, whereas at room temperature following a thermal cycle (see Sec. II C) $f_{d}$ is $45.2 \pm 0.1 \%$ with the laser on and $25.1 \pm 0.2 \%$ with the laser off.

One important difference between the current arrangement and that described previously [43] is that additional differential pumping sections between the positron trap and the target chamber have been added, as shown in Fig. 2. This was done in order to reduce sample exposure to the gases used in the positron trap $\left(\mathrm{N}_{2}\right.$ and $\left.\mathrm{CF}_{4}\right)$. The $\mathrm{SiO}_{2}$ and Ge targets were mounted on a closed-cycle helium refrigerator with a $1000 \mathrm{~K}$ high-temperature interface, as shown in Fig. 3. The sample stage was electrically isolated from the hightemperature stage using a sapphire disk, allowing the positron impact energy to be controlled via the sample bias. Because of this the actual range of sample temperatures was $\sim 20-750 \mathrm{~K}$. All quoted temperatures regarding the high-temperature coldhead are as measured using the Pt sensor attached to the hightemperature stage beneath the target mount. With the target grounded a K-type thermocouple was placed directly on the sample holder. A maximum temperature of $780 \mathrm{~K}$ was recorded at the target when the Pt sensor read $850 \mathrm{~K}$, indicating a large thermal gradient between the two for these high temperatures. For temperatures in the range of $27-700 \mathrm{~K}$ the sensors agreed to within $5 \mathrm{~K}$. Holes in the heat shield (see Fig. 3) raised the minimum achievable temperatures from $\sim 15 \mathrm{~K}$ to around $\sim 20 \mathrm{~K}$, which is also the lower limit of the Pt sensor range.

Some experiments were conducted using an alternate cold head with an operating range of 12-300 K, installed in both the current, and the previous a target chamber [43], which had poor vacuum isolation from the buffer gas trap. The pressure in this case was $\sim 2 \times 10^{-6}$ mbar during normal trap operation. Under these conditions, Ps formation in $\mathrm{SiO}_{2}$ samples was inhibited almost immediately after cooling to low temperatures (see Sec. III A).

\section{B. Laser systems}

Some of the experiments described here required intense pulses of 532-nm radiation to bombard semiconductor targets at the time of positron implantation in order to enhance the production of Ps. This laser radiation was obtained by frequency doubling the 1064-nm fundamental output of a Q-switched Nd:YAG laser, producing 532-nm laser pulses with a time width of $\sim 6 \mathrm{~ns}$ (FWHM). The areal profile of the beam was approximately Gaussian, with an area of $\sim 3 \mathrm{~cm}^{2}$, and an energy per pulse of $\sim 150 \mathrm{~mJ}$, giving average fluences 


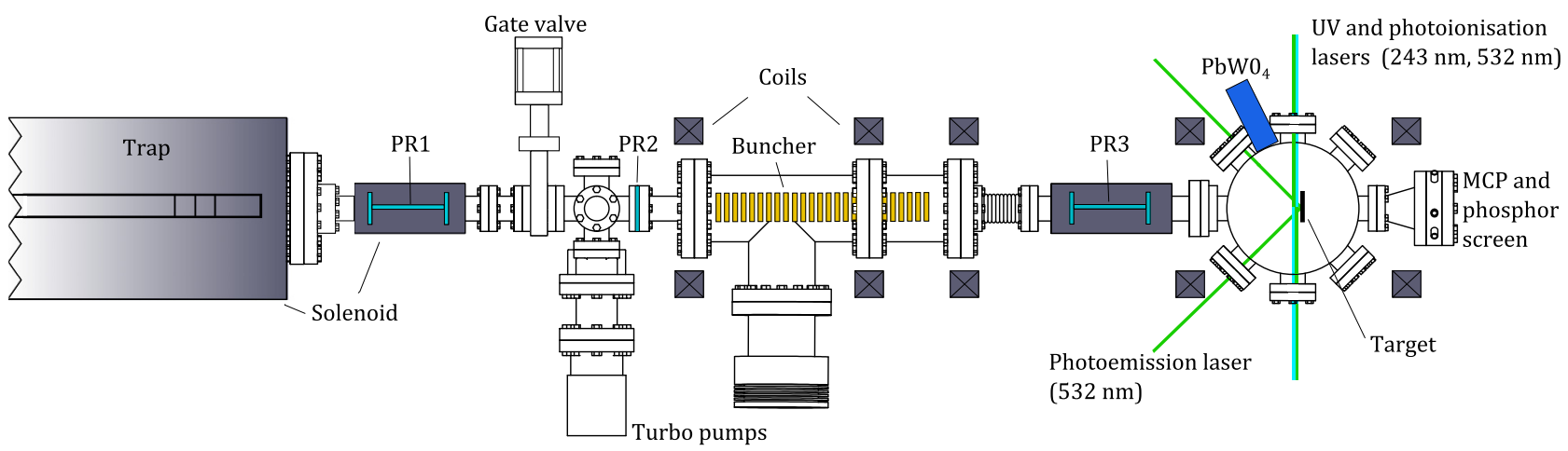

1 meter

FIG. 2. Schematic layout of the positron beam following the two-stage buffer gas trap. The ${ }^{22} \mathrm{Na}$ source and slow positron beam that precedes the trap are described in Ref. [43]. Differential pumping is achieved using two 8-mm diameter tubes located inside solenoids (PR1 and PR3), and a 10-mm aperture (PR2) as indicated in the figure. Positrons are ejected from the trap with a 30-ns time width (FWHM) and are further bunched in time by a pulsed parabolic potential to a width of $\sim 6 \mathrm{~ns}$ (FWHM) at the target. The configuration shown yields a pressure of $\sim 10^{-9}$ mbar in the main target chamber. The cold head was mounted on an $x y z$ manipulator and could be moved entirely out of the beam path, allowing the positron beam profile to be recorded on an imaging mirco-channel plate (MCP) detector and phosphor screen assembly. The $\mathrm{PbWO}_{4}$ scintillator is coupled to a PMT and oscilloscope in order to perform SSPALS [51].

of $\sim 50 \mathrm{~mJ} / \mathrm{cm}^{2}$, and peak powers of $\sim 24 \mathrm{MW}$. The fluence of the 532-nm radiation was controlled via a combination of neutral density filters and by tuning the angle of the KDP (potassium dihydrogen phosphate) nonlinear doubling crystal.

Some experiments required excitation of Ps from the ground state to $n=2$; this was achieved via the $1 \mathrm{~S}-2 \mathrm{P}$

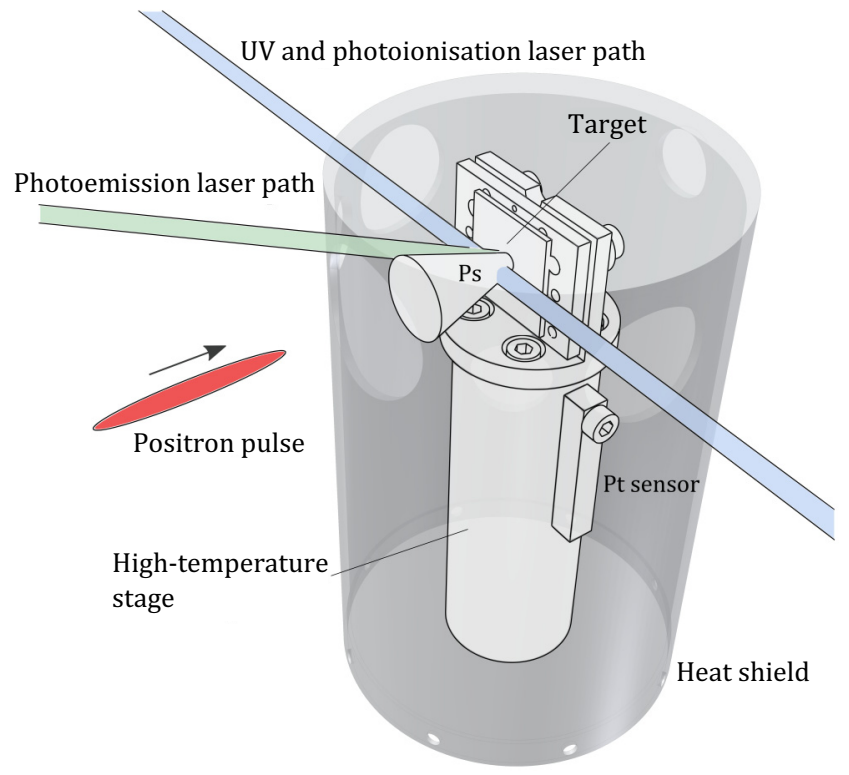

FIG. 3. Schematic layout of the high-temperature coldhead interface and positron beam and laser pathways. Holes are drilled in the heat shield to allow laser access and egress. The heat shield is at ground potential, and the target mount is biased to control the incident positron beam energy. transition, which requires ultraviolet (UV) radiation of wavelength $243.01 \mathrm{~nm}$. This radiation was obtained from a pulsed dye laser, pumped by the third harmonic of an additional Nd:YAG laser. The dye laser produced radiation of $486.02 \mathrm{~nm}$, which was frequency doubled to produce the desired 243.01-nm radiation. This pulsed dye laser was operated using a multiple-prism oscillator, producing laser radiation with a bandwidth of $\sim 85 \mathrm{GHz}$. The lasing efficiency of the dye, as well as the doubling efficiency, resulted in an output of $\sim 2 \mathrm{~mJ}$ per $6 \mathrm{~ns}$ (FWHM) pulse. The beam has a rectangular spatial profile of $\sim 2$ by $0.4 \mathrm{~cm}$, leading to average fluences of $\sim 2.5 \mathrm{~mJ} / \mathrm{cm}^{2}$ and peak powers of $\sim 313 \mathrm{~kW}$.

Experiments where Ps was excited to $n=2$ using 243.01-nm radiation also utilized a second 532-nm laser pulse to photoionize the $2 \mathrm{P}$ states as a means of detection. The second 532-nm laser was separated from the residual of the radiation used to generate the Nd:YAG's third harmonic (for pumping the dye laser). This radiation had a Gaussian profile, with an area of approximately $1 \mathrm{~cm}^{2}$, fluences of $\sim 30 \mathrm{~mJ} / \mathrm{cm}^{2}$ and peak powers of $\sim 5 \mathrm{MW}$.

\section{Sample preparation}

\section{1. $\mathrm{SiO}_{2}$}

The $\mathrm{SiO}_{2}$ samples were made using the sol-gel method [53], with TEOS (tetraethyl orthosilicate) as a precursor to silicon dioxide. The triblock copolymer Pluronic ${ }^{\circledR}$ F-127 (poloxamer 407) was used as a template, following the method of Yantasee et al. [54] with a higher porogen to silicon ratio (0.016 instead of 0.008 molar ratio). A mixture of F-127, TEOS, ethanol, deionized water and $\mathrm{HNO}_{3}$ in a molar composition of 0.016:1:8:8:0.05 was stirred for 90 minutes at room temperature. The film was deposited using spin coating at 
$3000 \mathrm{rpm}$ on a $p$-type silicon single crystal wafer. The porogen was removed from the film by heating the samples to $723 \mathrm{~K}$ for 15 minutes in air.

In the sol-gel preparation method, spherical micelles with a well defined size are formed by the porogen and the $\mathrm{SiO}_{2}$ walls are formed from the TEOS precursor. The micelles decompose in the annealing phase, leaving behind a pure $\mathrm{SiO}_{2}$ structure with a porous network defined by both the spherical micelles and the reduction of the film thickness. The pore surface exhibits silanol $(\mathrm{Si}-\mathrm{O}-\mathrm{H})$ groups with typically $4-5 \mathrm{OH} / \mathrm{nm}^{2}$ density. In addition, the presence of surface contamination from the decomposition products of the porogen can be expected when annealing at temperatures below $773 \mathrm{~K}$. These decomposition products are carbon based, mostly comprised of methoxyl $\mathrm{Si}-\mathrm{O}-\mathrm{CH}_{3}$ groups.

Earlier studies with similar samples found $31 \%$ Ps emission into vacuum with $2-4 \mathrm{keV}$ beam energies. As seen from the energy dependence of the vacuum $o$-Ps fraction, the fraction of positrons, which annihilate in the substrate is not significant below $4 \mathrm{keV}$ beam energies, and the $o$-Ps fraction remains constant in the $2-4 \mathrm{keV}$ positron energy range. The pore size in similar films with a lower porogen (F-127) ratio was found to be $7.7 \mathrm{~nm}$ by the BET (Brunauer, Emmett, Teller) method [54]. However, on the basis of positron lifetime measurements, a pore size of $4 \mathrm{~nm}$ was estimated [41]. The apparent discrepancy between the two values may be due to pore distortion caused by a higher porogen fraction than used by Yantasee et al. [54]. The porosity of the $\mathrm{SiO}_{2}$ samples used in this study was not directly measured, but we estimate it to be around $50 \%$.

\section{Single crystal Ge}

The semiconductor studies reported here were undertaken using a $p$-type $\mathrm{Ge}(100)$ single crystal. The dopant was $\mathrm{Ga}$ and had a manufacturer stated resistivity of $0.019-0.024 \Omega$. $\mathrm{cm}$. Additional measurements were performed using an $n$-type crystal (doped with $\mathrm{Sb}$, with a manufacturer stated resistivity of $0.01-0.1 \Omega . \mathrm{cm}$ ) but no significant differences between the two were observed, except that the photoemission process (see Sec. III B) from $p$-type Ge seemed to be more efficient, as was also observed in the previous measurements with $\mathrm{Si}$ [10].

The samples were delivered with polished surfaces in sealed containers. Before use they were immersed in distilled water for one minute to dissolve the native oxide layer, and then etched in $32 \% \mathrm{HCl}$ for 3.5 minutes. The samples were then dried with $\mathrm{N}_{2}$ gas and inserted into the vacuum chamber (within 20 minutes) where they were heated in order to desorb $\mathrm{Cl}$ terminated dangling bonds (see Sec. IIIB). All of the Ge data shown here were taken using $p$-type $\mathrm{Ge}(100)$ crystals.

\section{RESULTS AND DISCUSSION}

\section{A. Mesoporous $\mathrm{SiO}_{2}$}

It has been known for many years that Ps atoms are efficiently produced when positrons interact with fine powders of various oxides [1]. In these materials Ps is formed by interactions with the electrons produced by positron implantation [55]. Ps then diffuses to the grain surface [56] and is emitted with a kinetic energy that is related to the positron and electron work functions, the band-gap energy in the material, and the Ps binding energy [57]. In the case of $\mathrm{SiO}_{2}$, bulk Ps is emitted into vacuum with approximately $1 \mathrm{eV}$, although higher energies can be observed due to surface-based formation processes [58]. The initial Ps energy is reduced following collisions, but complete thermalization requires very deep implantation [36,37,59].

More efficient Ps thermalization can be achieved using Ps emitting insulators that have been assembled as thin mesoporous films $[60,61]$. These films, which can be formed from a wide variety of materials (see, for example, Ref. [9]), allow for efficient Ps formation and cooling [7,62]. The underlying Ps formation and cooling processes in such materials are similar to those of granulated powders [37,63], but the energy loss rates are generally faster. There is however an intrinsic limitation to the temperature of Ps emitted into vacuum from a mesoporous film that arises from the fact that the Ps de Broglie wavelength (approximately $6 \mathrm{~nm}$ at $300 \mathrm{~K}$ ) can become comparable to the mean pore size. When this happens Ps atoms are confined in the voids with a minimum (zero-point) energy that depends on the size of the cavity. Thus after moving through the porous network (or a subset thereof [64]) via a tunneling-limited diffusion process, Ps atoms may be emitted into vacuum with a nonthermal kinetic energy, determined by the quantum mechanical confinement energy $[41,49]$.

If the pores are made larger to reduce the confinement energy, Ps formation and cooling efficiencies are expected to be reduced. There are minimal experimental data on the effects of the pore size on the Ps formation efficiency in mesoporous $\mathrm{SiO}_{2}$. In all mesoporous films Ps is formed efficiently in the bulk material, where typical lifetimes are sub-nanosecond, extending to a few nanoseconds if micropores are present [8]. Therefore, in order for Ps atoms to be emitted into vacuum they must be created in close proximity to a void, which is more likely for converters with smaller pores and higher porosity, and less likely in the case of larger pores, where the average wall thickness is higher. The underlying assumption that the microstructure of the $\mathrm{SiO}_{2}$ walls separating the pores is the same in all these structures is not necessarily correct. A network of micropores may be formed around the mesopores [65], which is transparent for the diffusing Ps. This can lead to efficient Ps production in a part of the walls between the pores. Ps created in the micropores then diffuse to the mesopores and increase the overall Ps emission. Nevertheless, lower Ps emission efficiencies have been observed in macroporous silica films with pore sizes of 32-70 $\mathrm{nm}$ [66], and somewhat higher in mesoporous films with smaller pores [62], supporting the expectation that the Ps production efficiency increases with decreasing pore size.

The most efficient mesoporous Ps converters currently available have pore sizes of around $5 \mathrm{~nm}$ or less [41]. These produce Ps with near-thermal energies (or above, due to the confinement energy problem). Research is currently underway with the objective of producing lower energy Ps atoms without loss of efficiency. We note that the confinement energy limitation can in principle be mitigated by using long nanochannels rather than mesoporous films $[17,67]$.

Since the underlying production mechanism that occurs in mesoporous Ps converters is not thermal, they can be 


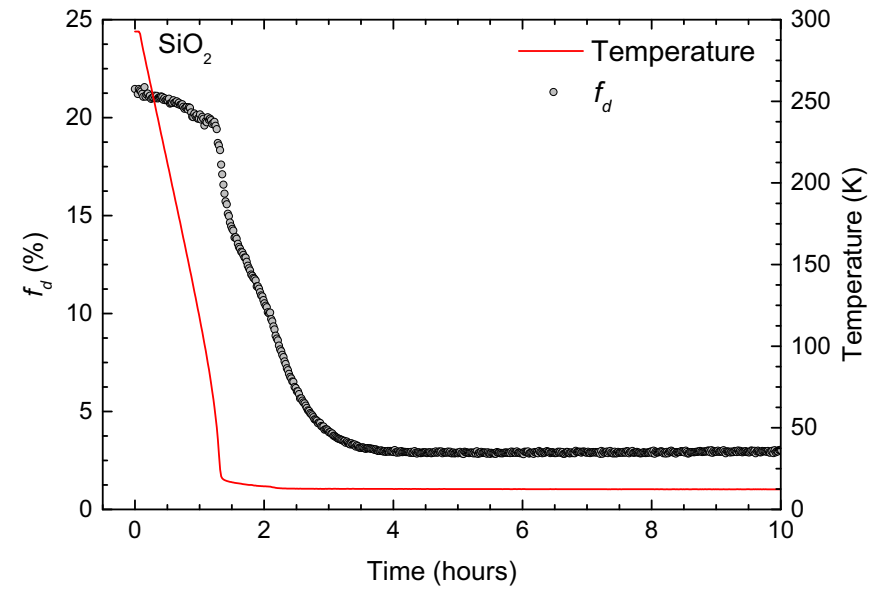

FIG. 4. Delayed fraction $f_{d}$ from cooled mesoporous $\mathrm{SiO}_{2}$ measured in the high-pressure $\left(5 \times 10^{-6} \mathrm{mBar}\right)$ chamber. The delayed fraction error bars $( \pm 0.1 \%)$ are not shown.

expected to work with similar efficiency at any temperature. In mesoporous materials, as well as metal oxide powders, Ps atoms lose their energy primarily via inelastic collisions, and must have hundreds of thousands of surface or grain interactions in order to thermalize $[8,68]$. Therefore they are very sensitive to the surface conditions, including adsorbed surface material. In some cases such contamination may be advantageous, leading to an increased cooling rate [69], but more often it merely leads to a reduction in the amount of Ps emitted [70,71]. This effect is explicitly demonstrated in Fig. 4, which shows Ps formation in a chamber with inadequate vacuum isolation from the buffer-gas trap. These data show that when the chamber pressure is around $5 \times 10^{-6}$ mbar, Ps formation is significantly reduced within a few hours of the target being cooled down $\left(f_{d} \sim 4 \%\right)$. The reduction in $f_{d}$ is attributed to the freezing of $\mathrm{N}_{2}$ and $\mathrm{CF}_{4}$ gas from the positron trap. The target recovers completely after heating back to room temperature.

The adsorption of gas molecules in the porous matrix can affect Ps lifetimes, and hence reduce the emission efficiency, simply by reducing the available free volume. The use of Ps as a probe of porous materials has been highly successful in part because Ps-wall interactions have proved to be largely insensitive to the particular materials studied [72]. This allows meaningful correlations between Ps lifetimes and pore sizes to be applied to samples made from a wide range of materials using only calibrations related to the physical structure of the pore network. We attribute the reduction of Ps emission observed after gas absorption primarily to a reduction in the available pore volume. For smaller effective pores, the rate of Ps-wall interactions increases, reducing the Ps lifetime. It should be remembered that, since Ps is initially formed in the bulk, condensed gas in porous films does not reduce the amount of Ps that is initially created. Rather, it reduces the Ps lifetime in the pore network so that fewer atoms are emitted into vacuum.

Problems arising from gas absorption can of course be reduced or eliminated by improving the vacuum quality. Figure 5 shows $f_{d}$ measured in a vacuum chamber where the base pressure is $\sim 10^{-9}$ mbar. In this case, $f_{d}$ again decreases with time, but at a much slower rate, dropping by almost $10 \%$

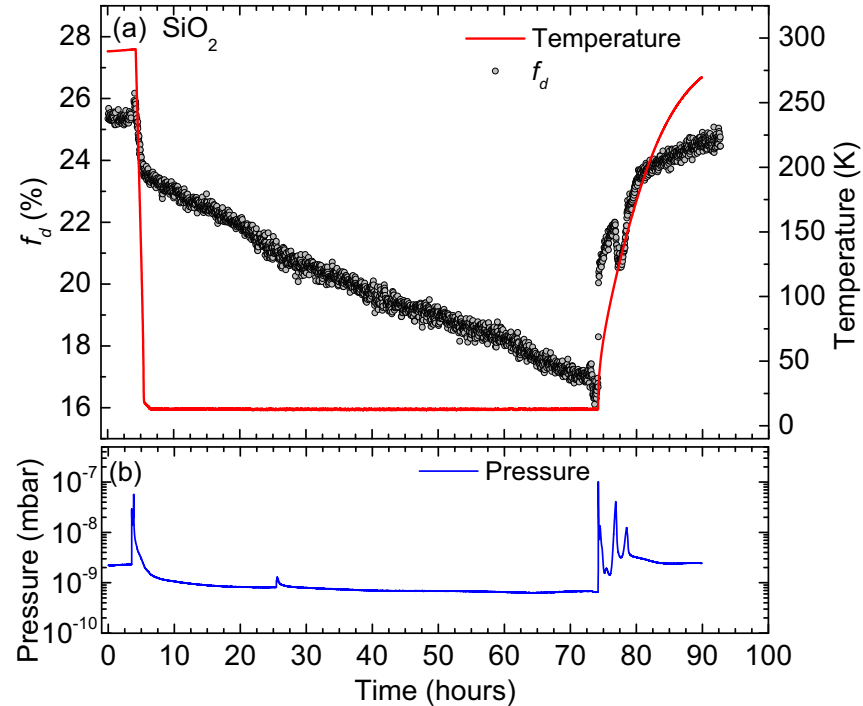

FIG. 5. (a) Delayed fraction $f_{d}$ from cooled $\mathrm{SiO}_{2}$ measured in the low-pressure $\left(10^{-9} \mathrm{mBar}\right)$ chamber. (b) Pressure in the target chamber associated with the cooling and heating cycle of (a).

per day after an initial drop of $\sim 15 \%$. The reduction in $f_{d}$ is attributed to the adsorption of residual gas in the system, mostly $\mathrm{N}_{2}, \mathrm{CF}_{4}$, and $\mathrm{H}_{2} \mathrm{O}$. The recovery and subsequent structure observed in the warm up curve is attributed to the desorption of these gases at different temperatures, as evidenced by the fluctuations in the chamber pressure.

It has previously been observed that neither the production efficiency nor the kinetic energy of Ps emitted from $\mathrm{SiO}_{2}$ depend strongly on the sample temperature within the range of 50-300 K [41]. We confirm this observation here via Doppler spectroscopy of Ps [49] emitted from $\mathrm{SiO}_{2}$, as shown in Fig. 6. These data show Gaussian fits to the Doppler broadened lineshapes yielding $\sigma$ of $0.070 \pm 0.001 \mathrm{~nm}$ and $0.069 \pm 0.002 \mathrm{~nm}$ for sample temperatures of 300 and $12 \mathrm{~K}$. This corresponds to

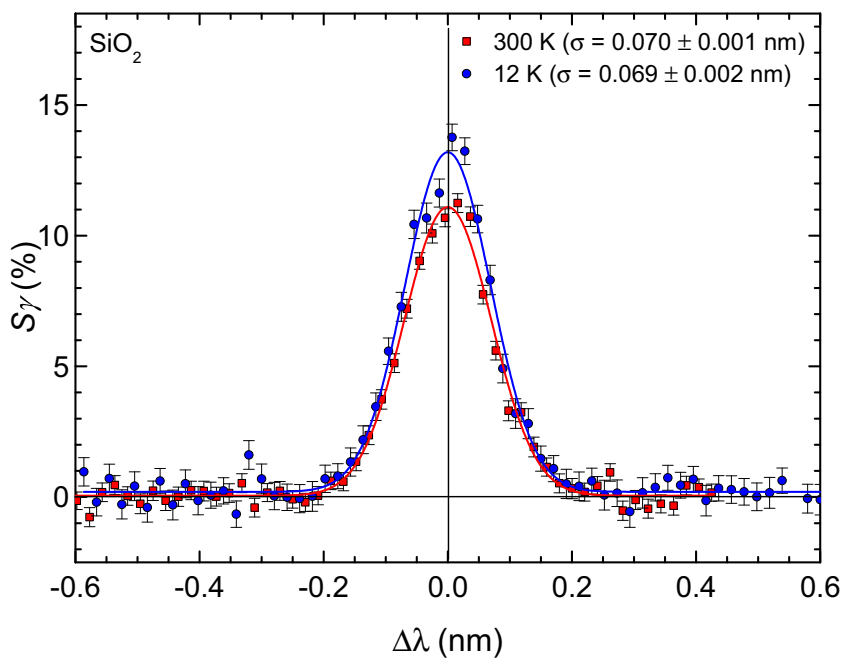

FIG. 6. Doppler broadened 1S-2P line-shape measurements at 300 and $12 \mathrm{~K}$. The central wavelength $(\Delta \lambda=0)$ is $243.01 \mathrm{~nm}$. The data are fitted to Gaussian functions. The values of the Gaussian width $\sigma$ are shown in the legend. 
Ps kinetic energies of around $40 \mathrm{meV}$ along the direction of the laser path, which is parallel to the $\mathrm{SiO}_{2}$ sample surface.

Ps porosimetry [72,73] is based upon the fact that Ps atoms in mesoporous films can collide with internal pore surfaces hundreds of thousands of times without annihilating. During these collisions, the annihilation rate is approximately the spin averaged decay rate weighted by the 3:1 triplet-singlet ratio [8] $\left(\sim 2 \mathrm{~ns}^{-1}\right)$. In the quantum mechanical version of this model, the spin-averaged decay rate is applied only to the portion of the wave function deemed to interact with the wall (which is usually determined empirically). In either case, the decay rate due to Ps-wall interactions is small, so that the total decay rate may be comparable to the vacuum rate for sufficiently large pores. However, if there are paramagnetic centers present the Ps decay rate will increase significantly due to spin conversion processes [74,75].

A paramagnetic center is any complex in an insulator that has an unpaired spin, and there are many varieties. The experiments described in this work cannot distinguish between different paramagnetic species. In order to do so, it would be desirable to perform combined Ps lifetime and electron paramagnetic resonance (EPR) techniques. This has been done in previous experiments [76] using bulk oxide grains (including silica) but has not yet been demonstrated with thin films. Since Ps interactions in mesoporous films take place predominantly on internal surfaces, using time-resolved annihilation and EPR methods [77] could provide new insights into transient or laser-induced surface reactions. Furthermore, identifying the exact types of paramagnetic centers that inhibit Ps formation may help to produce Ps converters that are less sensitive to radiation damage at low temperatures.

The effects of various paramagnetic centers on Ps atoms have been extensively studied in different materials [76,78-81]. Positron irradiation is itself able to generate paramagnetic centers [80,82-84], but irradiation with UV photons is far more efficient. The experiments of Saito and co-workers $[76,80]$ have demonstrated conclusively that paramagnetic centers produced by UV light can significantly affect the lifetime of Ps atoms. Most of these centers are not stable at room temperature, and will recombine almost instantaneously via thermal fluctuations. However, at cryogenic temperatures they may become very stable, with extremely long recombination times. Our measurements, shown in Fig. 7 , confirm this observation; irradiation of an $\mathrm{SiO}_{2}$ sample cooled to $12 \mathrm{~K}$ with $10 \mathrm{~Hz}$ UV laser pulses reduced $f_{d}$ substantially. When kept at low temperature following irradiation [Fig. 7(c)], no time-dependent recovery was observed. Recovery to the initial value of $f_{d}$ (prior to cooling and laser irradiation) was observed only after the sample was heated to room temperature [Fig. 7(a)].

The data shown in Fig. 7(a) suggest that there may be two different types of paramagnetic center present, as indicated by the temperature dependence of the recovery. If the reduction in $f_{d}$ were due to a single type of isolated paramagnetic center with a well-defined activation energy then we might expect to see threshold behavior in the recovery curve. However, if there are many different local environments, or types of paramagnetic center, the activation energies for thermal recombination may be spread over a quasicontinuum. These data show explicitly that laser irradiation results in
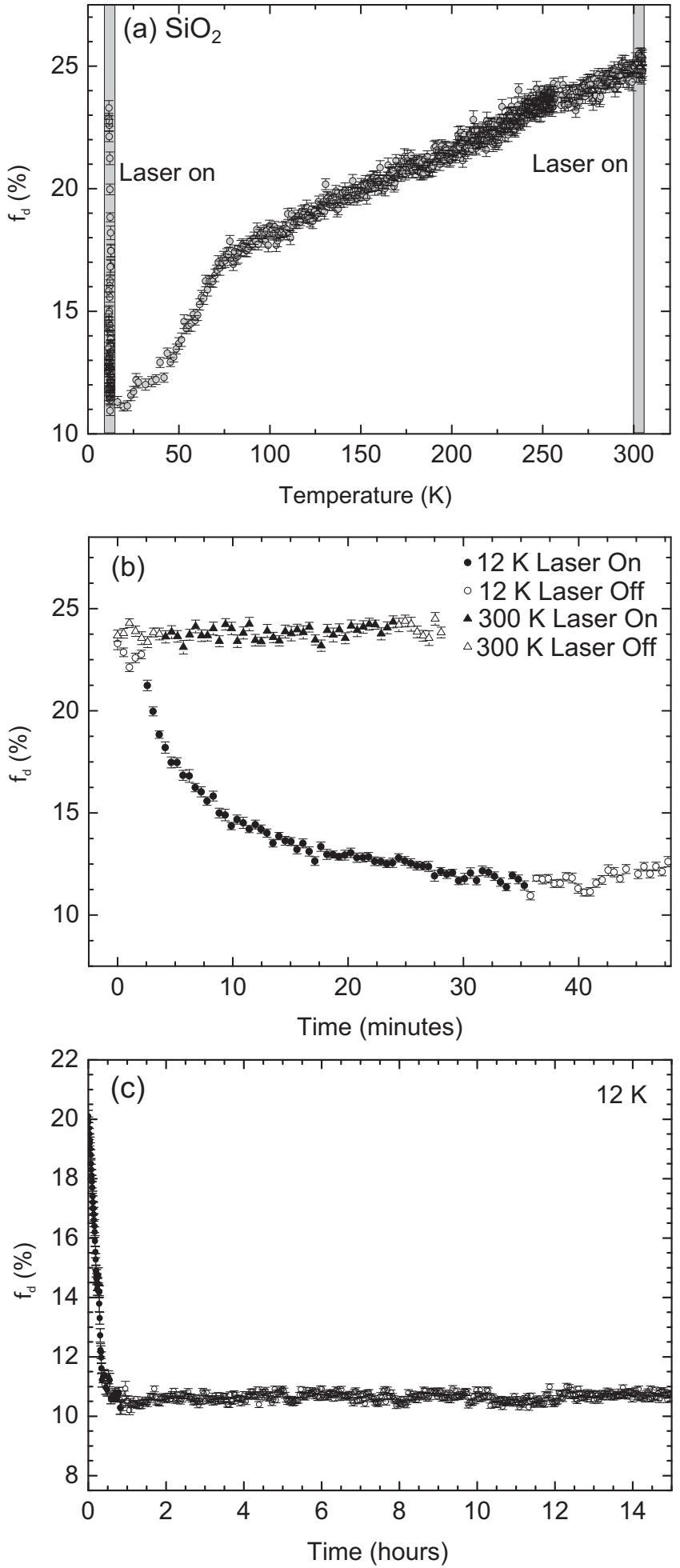

FIG. 7. (a) Delayed fraction $f_{d}$ measured as a function of temperature for an $\mathrm{SiO}_{2}$ sample irradiated with UV light pulses applied at $10 \mathrm{~Hz}$. (b) Data from (a) plotted against time for laser irradiation at 12 and $300 \mathrm{~K}$, as indicated in the legend. (c) Long-term stability of laser-induced paramagnetic centers. The sample remains cold $(\sim 12 \mathrm{~K})$ following irradiation.

the production of stable paramagnetic centers only when the sample is cooled below room temperature [in particular Fig. 7(b)]. 
In the experiments of Saito and Hyodo [76], no radiation induced effects on Ps lifetimes were observed in silica powder or silica aerogel that had been heat treated to $1073 \mathrm{~K}$. This was attributed to the formation of $-\mathrm{OCH}_{2}$ radicals, which were identified via electron paramagnetic resonance measurements. In the present experiments, we were not able to check if heating the targets to similar temperatures would eliminate the radiation-induced effect owing to the operating range of the high-temperature interface. We did, however, observe an increase in $f_{d}$ from a mesoporous $\mathrm{SiO}_{2}$ film that had been heated to $600 \mathrm{~K}$ in situ, as shown in Fig. 8(a). The increased yield was mostly retained when the sample was cooled back to room temperature. Two possible explanations for the improved yield are the desorption of material from the porous network, and the induced recombination of paramagnetic centers that are stable at room temperature. The data in Fig. 8 suggest a combination of both.

Figure 8(a) shows the heated sample returning to room temperature and the measured Ps yield, which was only slightly reduced from the maximum value observed at $600 \mathrm{~K}$. This rules out an effect due to thermal desorption of Ps from surface states, which have been observed previously in both quartz targets [58] and in silica films [85]. After heating, the $\mathrm{SiO}_{2}$ sample became susceptible to a laser-induced reduction in the Ps yield [see Fig. 8(c)], strongly suggesting the production of stable paramagnetic centers. This irradiation did not, however, return the sample to its previous state, suggesting that either some of the improvement may have been due to desorption of material, or possibly that the stable paramagnetic centers are more difficult to populate. Such centers have been previously observed using capped mesoporous films [86]. If these stable paramagnetic centers were already present at a saturated level in a sample that had not been heated then we would not expect to see any effect of laser irradiation, as shown in Fig. 7.

When the heated sample was cooled to $27 \mathrm{~K}$ and irradiated, a further reduction in $f_{d}$ was observed, as shown in Fig. 8(b). The saturated $f_{d}$ value in this case was slightly higher than was observed for the unheated sample, but we would expect further decline with time. Thus any benefits obtained from heating do not appear to survive in cryogenic conditions, but they can be recovered if the sample is again warmed up to $\sim 600 \mathrm{~K}$, as shown in Fig. 8(b).

Measurements of $f_{d}$ for different positron implantation energies and sample temperatures are shown in Fig. 9. The target bias was scanned at each of the stated temperatures in ascending order. The data shown in Fig. 9(a) were recorded with no previous laser irradiation, whereas those shown in Fig. 9(b) were recorded following laser irradiation at $27 \mathrm{~K}$ until the reduction in $f_{d}$ reached saturation. Varying the target bias across the range shown in Fig. 9 took approximately 25 minutes for each temperature, therefore only minimal gas adsorption effects are expected [see Fig. 5(a)]. These data indicate the location of laser induced paramagnetic centers in the target, since Ps formation occurs at a depth determined by the incident positron beam energy and implantation profile [87-89]. The approximate mean depth of the incident positron beam is indicated on the top axis of Fig. 9(a). The exact thickness of the present film is unknown, but is estimated to be at least $500 \mathrm{~nm}$. The lack of any sharp features at high
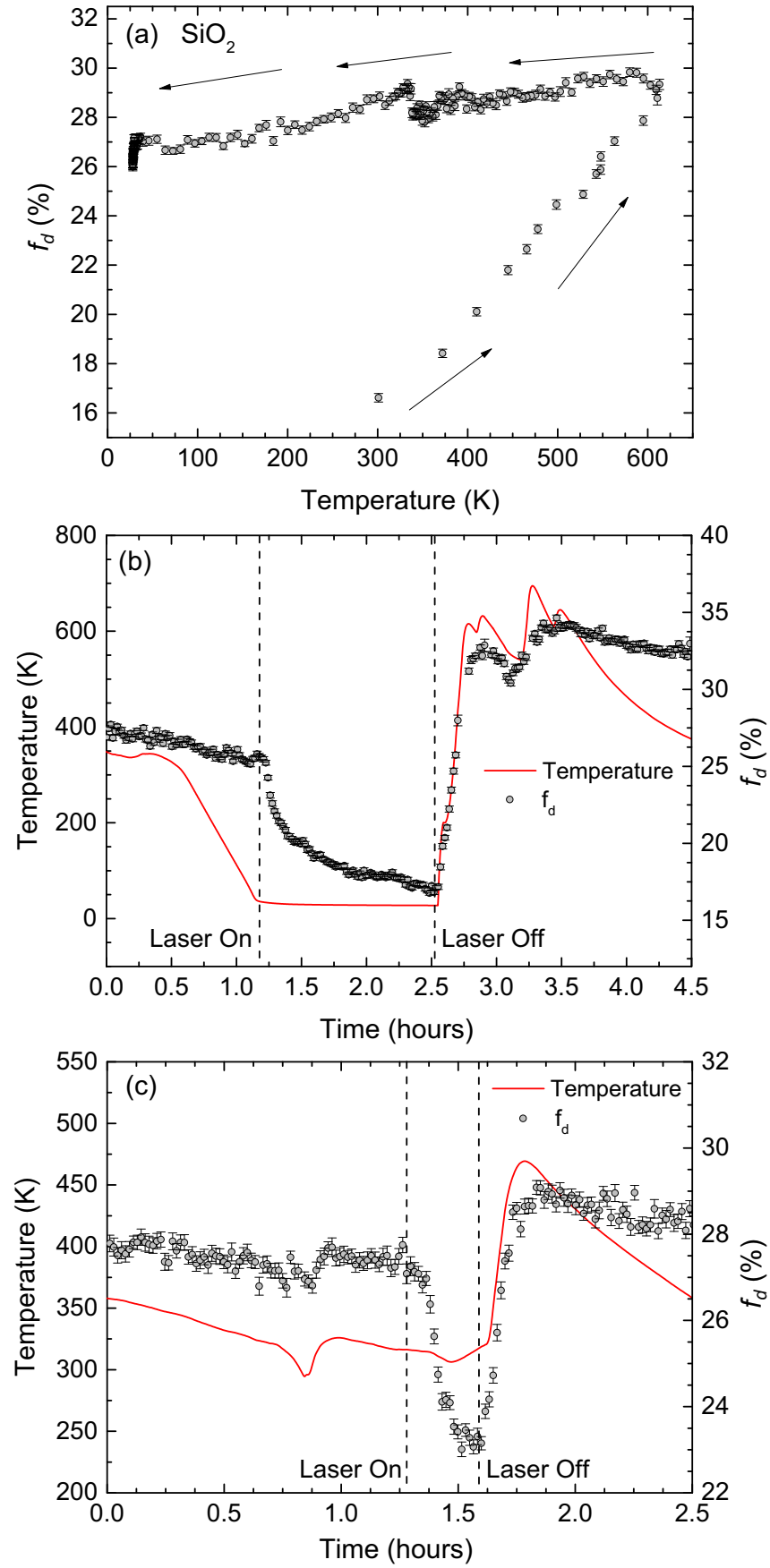

FIG. 8. (a) Delayed fraction $f_{d}$ (filled circles) measured as a function of temperature for an $\mathrm{SiO}_{2}$ sample as it is heated. Each point represents the average $f_{d}$ value over a 30 second period. (b) Effect of cooling and laser irradiation on the delayed fraction, and (c) laser irradiation at room temperature following heating to $\sim 600 \mathrm{~K}$. In (b) and (c), the sample temperature is indicated by the solid (red) lines.

implantation energies in Fig. 9 indicates that the film thickness is always greater than the positron implantation depth.

In general, we expect a decrease in $f_{d}$ as the positron beam energy is increased because Ps will then have to diffuse further through the pore network before emission. However, with the present samples we expect only a negligible decline 


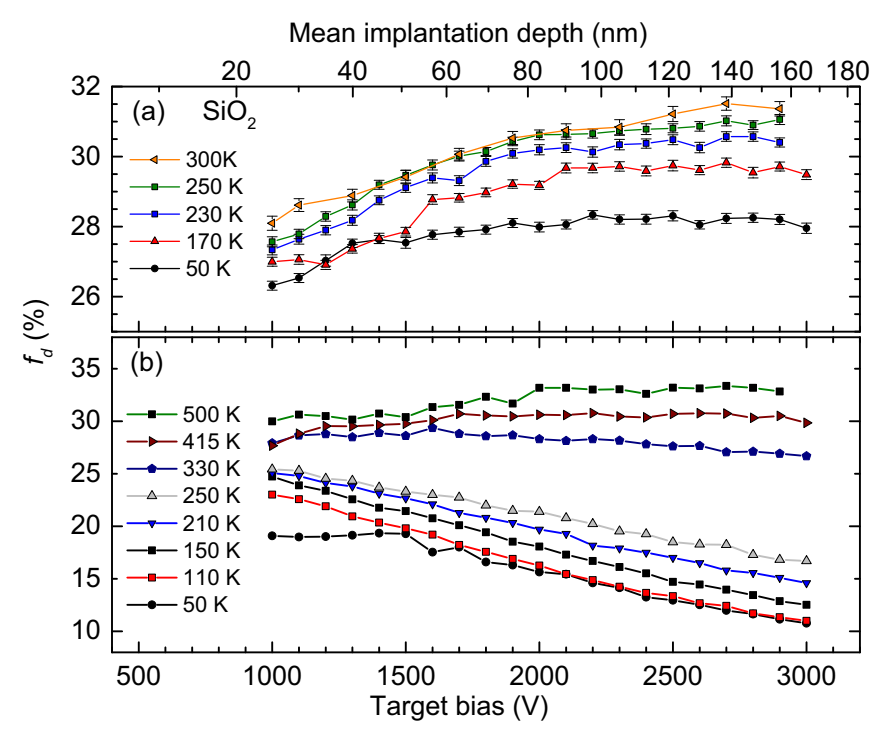

FIG. 9. $f_{d}$ vs target bias at various temperatures for an $\mathrm{SiO}_{2}$ sample with (b) and without (a) prior UV laser irradiation. The top axis indicates the mean implantation depth of the positrons. The irradiation and heating sequence is explained in the text. Error bars are not shown in (b) but are a similar size to those in (a).

for implantation energies of $3 \mathrm{keV}$, in accord with previous measurements [41]. In fact, we observed a small increase (less than $\sim 10 \%$ ) in the values of $f_{d}$ when the beam was implanted at higher energies in Fig. 9(a). This effect is sometimes observed when fast Ps is able to move away from the $\gamma$-ray detector [90].

For the irradiated target [Fig. 9(b)], we observe no energydependent reduction in $f_{d}$ when the sample temperature is above $250 \mathrm{~K}$, in agreement with the model of temperature stabilized paramagnetic centers. At these temperatures, we predict that the laser would have no effect, and changes in $f_{d}$ due to the beam energy increasing would be similar to those observed in a target that had not received laser irradiation. At lower temperatures $f_{d}$ is reduced by around $50 \%$ at the higher implantation energies. This indicates that either the paramagnetic centers that affect Ps atoms are created predominantly in the deeper layers of the target, or that they are primarily on the internal surfaces of the pores. Our data suggest the latter, as do direct lifetime measurements performed on irradiated silica powders [76].

Most of the incident UV light is reflected from the target substrate [91], suggesting uniform irradiation of the $\mathrm{SiO}_{2}$ film and the creation of paramagnetic centers throughout. Since Ps formation occurs in the spur electron cloud [56], bulk paramagnetic centers probably do not affect Ps formation. The fact that Ps emission is inhibited by interactions with surface paramagnetic centers is known from previous irradiation measurements conducted with a capped silica target that did not allow Ps atoms to escape into vacuum [86]. Ps produced deeper in the $\mathrm{SiO}_{2}$ will undergo more surface collisions before emission. The presence of surface paramagnetic centers is therefore consistent with the observed dependence on the positron implantation energy. However, the linearity of this dependence with the positron implantation energy is not trivially explained. It may be related to the way in which

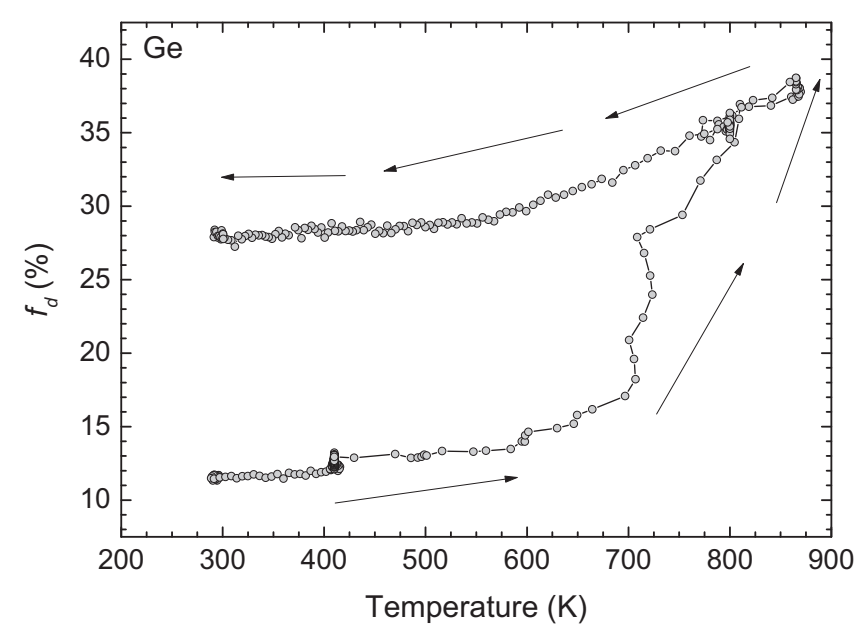

FIG. 10. Thermal desorption of $\mathrm{Cl}$ and concomitant increase in $f_{d}$, measured from single-crystal Ge after insertion into the vacuum system. The as-measured temperatures above $700 \mathrm{~K}$ are known to be inaccurate, as explained in the text. Each point represents the average $f_{d}$ value over a 30 second period.

confined Ps atoms move between pores [64]. This observation is broadly consistent with previous measurements of the Ps emission time from similar samples [50], which also suggested a linear dependence on beam energy. The observed energy dependence means that one can at least partially mitigate the effects of laser irradiation in cryogenic films simply by reducing the positron implantation energies, although this would result in the production of hotter Ps atoms [49].

\section{B. Single-crystal Ge}

After etching with $\mathrm{HCl}, \mathrm{Ge}$ crystal dangling bonds are expected to be terminated with $\mathrm{Cl}$, which inhibits Ps formation [10]. The $\mathrm{Cl}$ can be removed by heating the crystal, as shown in Fig. 10, in which an increase in $f_{d}$ is observed as the temperature is raised above $\sim 700 \mathrm{~K}$. Further heating of the $\mathrm{Ge}$ resulted in a higher Ps yield until a plateau was reached at the maximum temperature of $850 \mathrm{~K}$. As explained in Sec. II A, these measured high temperatures do not correspond to the true target temperature, and the actual maximum temperature was closer to $\sim 750 \mathrm{~K}$. The reasons for the apparent structure in the heating curve are not known, but may be caused by the dynamics of the surface cleaning process, or perhaps by out-gassing from the heater. This type of structure is not generally observed with Ge after the initial cleaning cycle.

Ps can be emitted from single crystal semiconductors via excitonlike positron-electron surface states [18]. Electrons that are excited into the conduction band may scatter into unoccupied surface states where they can form surface excitons by combining with holes in the surface band [92]. However, if positrons are present, electrons may combine with them instead and form Ps atoms. These exciton and Ps states have previously been denoted as $X$ [92] and Ps $X$ [42], respectively. Promoting an electron into the conduction band can be achieved thermally [18], or following interactions with photons [42]. We refer to the process by which light increases the Ps yield (through electron surface-state population) as photoemission 


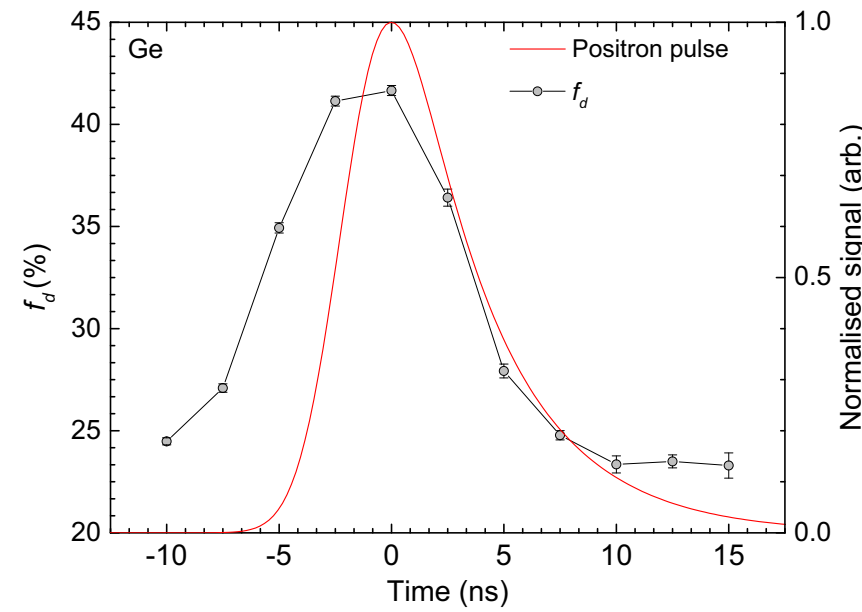

FIG. 11. Time dependence of Ps $X$ photoemission from a p-type $\mathrm{Ge}(100)$ crystal. The laser fluence was $\sim 50 \mathrm{~mJ} / \mathrm{cm}^{2}$. The solid (red) line is the positron annihilation signal measured with a fast $\gamma$-ray detector. This signal is the convolution of the prompt annihilation radiation and the $\sim 4 \mathrm{~ns}$ (FWHM) detector response [43].

[42]. We have achieved photoemission in Ge(100) samples using 532-nm (visible, green) laser irradiation. The Ps formation enhancement is most effective when the laser arrives at around the same time as the positron pulse, as shown in Fig. 11. The effect is still significant when the laser arrives up to $\sim 5 \mathrm{~ns}$ before the positrons indicating the lifetime of the surface states that participate in Ps $X$ formation. These surface states appear to have shorter lifetimes than those previously observed with Si targets [42], which persisted for many tens of nanoseconds.

An important question concerning the production of Ps from $\mathrm{Ge}$ in cryogenic environments is whether or not Ps $X$ can be produced via photoemission at low temperatures. Because Ps production from $\mathrm{Ge}$ is a surface process, it is much more susceptible to contamination from gas adsorption. Figure 12 shows $f_{d}$ measured from a cooled Ge sample with a range of laser fluences. It is evident from these data that the efficacy of Ps $X$ production is severely compromised at low temperatures

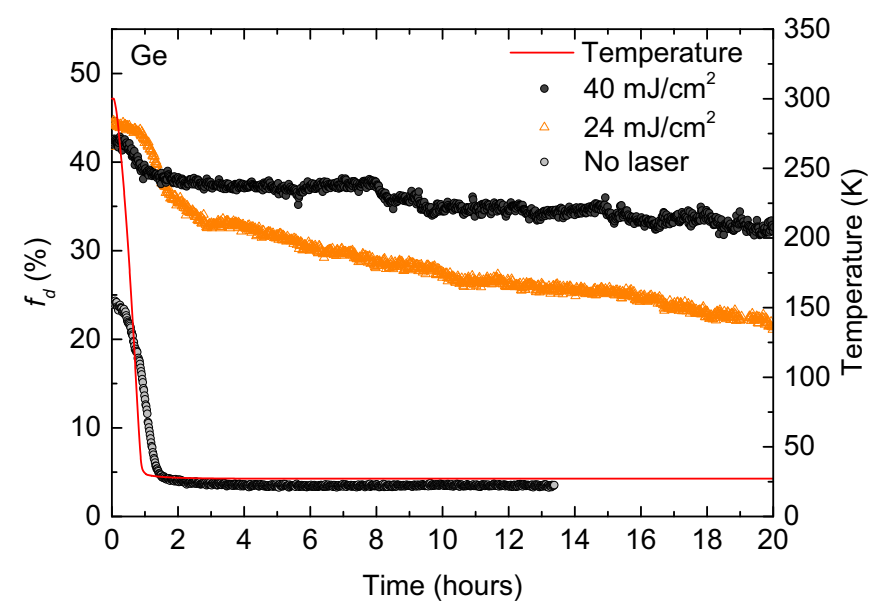

FIG. 12. Delayed fraction $f_{d}$ measured from a $p$-Ge(100) single crystal as the sample is cooled with and without 532-nm laser-induced photoemission. The laser fluence is indicated in the legend.

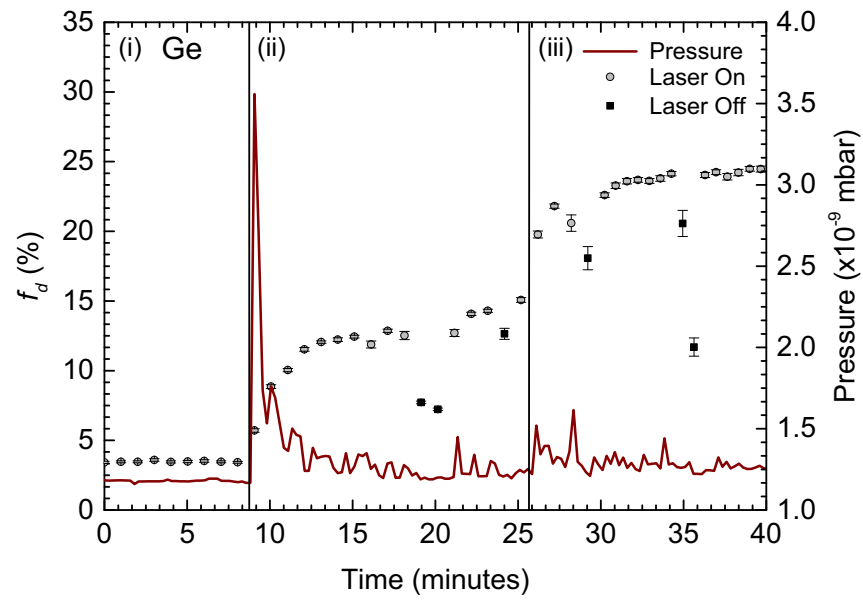

FIG. 13. Laser irradiation of a cold and contaminated Ge sample (the sample was kept at $27 \mathrm{~K}$ for $\sim 12$ hours) and its effect on $f_{d}$. Different laser fluences were tested, as indicated by the numbered regions $\left[(\right.$ i $)=$ laser off, (ii) $=10 \mathrm{~mJ} / \mathrm{cm}^{2}$, (iii) $\left.=24 \mathrm{~mJ} / \mathrm{cm}^{2}\right]$. The square points were recorded with the photoemission laser temporarily blocked.

without a photoemission laser, and that this occurs on a time scale much faster than that of $\mathrm{SiO}_{2}$ in the same vacuum [cf. Fig. 5(a)]. However, the Ps $X$ yield, and hence $f_{d}$, increase significantly when a photoemission laser is present. The efficiency and stability increase with the laser fluence. The data in Fig. 12 therefore show conclusively that Ps $X$ photoemission can be implemented at cryogenic temperatures. However, it is possible that the long-term stability of Ps $X$ emission could be compromised by the accumulation of residual gas on the crystal surface.

Figure 13 shows $f_{d}$ measured using a $p$-type $\operatorname{Ge}(100)$ target that had been cooled to $27 \mathrm{~K}$ for 14 hours. This results in essentially no emitted Ps (typical background levels are $3 \%-4 \%$ ). When the photoemission laser is present the signal is restored; recovery from $\sim 4$ to $25 \%$ is observed, indicating that the laser desorbs accumulated gas layers from the $\mathrm{Ge}$ surface. This is supported by the spike in the chamber pressure associated with the laser light (at around 9 minutes). The corresponding increase in the Ps yield, is consistent with both the desorption of surface gas layers and photoemission. Subsequent measurements with the laser blocked (black squares in Fig. 13) demonstrate the surface cleaning effect in isolation. Contamination occurs fairly quickly when the laser is blocked, but a relatively high Ps yield can be maintained when the laser is present. Even at the highest laser fluence the yield is somewhat less stable than is observed for thermal production. This is most likely due to the equilibrium between gas adsorption and desorption on the surface. There were no visible signs of any laser induced damage to the samples following long periods of irradiation.

Previous experiments with both $\mathrm{Si}$ and $\mathrm{Ge}$ showed that the energy of Ps $X$ is essentially independent of the sample temperature, whether it is generated by heating or by photoemission [10]. In fact, Ps $X$ emitted from very hot targets was slightly colder than that emitted from room-temperature targets (or via photoemission), which was attributed to shifts of the surface 

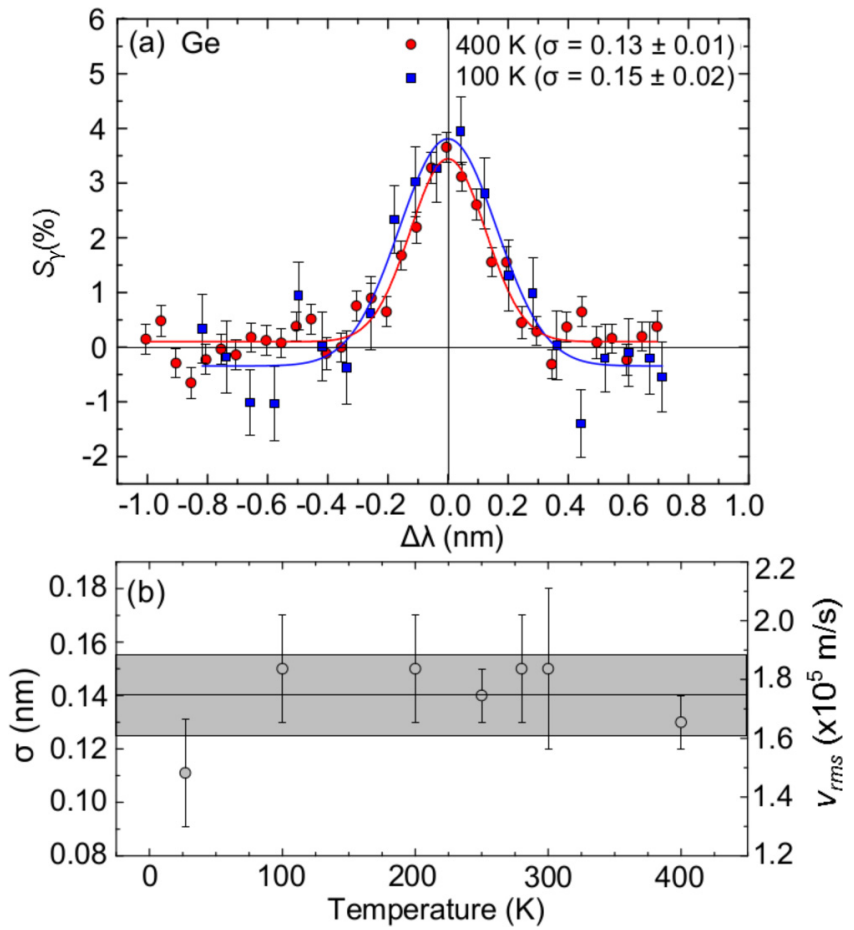

FIG. 14. Doppler spectroscopy of Ps emitted from single-crystal $\mathrm{Ge}(100)$. (a) Doppler broadened line-shapes measured at 400 and $100 \mathrm{~K}$. (b) Values of $\sigma$ obtained from linewidth measurements conducted at different sample temperatures. The gray band indicates the mean value of $\sigma \pm 1$ standard deviation $(0.140 \pm 0.015 \mathrm{~nm})$, and the corresponding RMS velocities. The positron beam energy was $0.65 \mathrm{keV}$ for the data shown in (a) and $0.5 \mathrm{keV}$ for the data in (b).

energy levels [10]. By measuring the Doppler-broadened 1S-2P transition linewidths, we have verified that the energy of Ps $X$ emitted via photoemission from cryogenic targets does not depend strongly on the target temperature. The $2 \mathrm{P}$ levels were detected following photoionisation with 532-nm light (generated independently of the photoemission laser light). No difference in the Ps energy was observed, as can be seen in Fig. 14, which shows Ps energies of around $170 \mathrm{meV}$ in the direction of the laser, for temperatures ranging from 27-400 $\mathrm{K}$.

The Ps $X$ kinetic energy is determined by the surface electron and positron energy levels, but the origin of the observed width of the Ps $X$ distributions is unknown. One possible explanation is that the thermally or optically excited surface electrons form Ps atoms before thermalizing. However, we do not believe this to be likely as the relevant electronic relaxation rates are in general very rapid [93,94]. Another possibility is that, if the Ps $X$ emission process involves surface interactions (e.g., phonon scattering), then even if the underlying energy levels are intrinsically narrow, atoms may nevertheless be emitted with a distribution of energies. However, the fact that the Doppler widths of Ps $X$ emitted from Ge at different temperatures (see Fig. 14) are the same indicates that thermally generated surface interactions do not in fact play a significant role in determining the Ps $X$ energy. If the crystal surface is not well-defined, then the electron and positron surface-state energies may vary from site to site, which could also give rise to variations in the emission energies. Similarly, there may be a wide range of available surface states with different energies, which would also lead to observations of a broad distribution.

It seems likely that the effects discussed here for $\mathrm{Ge}(100)$ would also apply to Si [10], and any other indirect band-gap semiconductor with dangling-bond states. If a material can be identified that exhibits photoemisison of Ps $X$ and has intrinsic energy levels lower than those of $\mathrm{Si}$ or $\mathrm{Ge}$, it may provide an efficient source of cold Ps atoms that can be used in cryogenic environments. This would be extremely beneficial for any experiments involving Ps laser excitation. For example, Doppler broadening is presently the limiting factor in the efficiency of 1S-2P excitation in Ps. Using typical near-thermal Ps sources, the linewidths of these transitions are generally broadened to more than $500 \mathrm{GHz}$ [49], requiring broadband pulsed light sources for efficient Ps excitation.

It is possible to obtain suitable solid-state UV lasers with large $(\sim 200 \mathrm{GHz})$ bandwidths [95], and if prisms [43,96] or high-order gratings [49] are used as dispersive media, it is relatively straightforward to produce light from dye lasers with bandwidths of around $100 \mathrm{GHz}$. However, improving the spectral overlap of an excitation laser with a given Ps transition by reducing Doppler broadening is preferable to using large bandwidth light sources. This is because considerably less power is required, and more accurate state selection becomes possible [97]. In addition, transit-time broadening and secondorder Dopper shifts are reduced if colder atoms are used.

\section{CONCLUSIONS}

Using a trap-based pulsed positron beam and singleshot lifetime spectroscopy we have measured Ps formation from mesoporous $\mathrm{SiO}_{2}$ films and $\mathrm{Ge}(100)$ single crystals at temperatures ranging from $12-700 \mathrm{~K}$. Our measurements show that Ps atoms can be created in cryogenic environments using both materials. The efficiency of production at cryogenic temperatures is comparable to that observed at room temperature, but can be compromised by residual gas contamination. For $\mathrm{SiO}_{2}$ films in a vacuum of around $10^{-9}$ mbar, the Ps formation decays on a time scale of many days, and can be restored by heating the sample to room temperature. However, these samples will decay rapidly if they are directly exposed to UV laser light. This is due to the creation of paramagnetic centers that become stable at cryogenic temperatures $[76,86]$. Warming cold irradiated $\mathrm{SiO}_{2}$ films to room temperature completely restores the Ps formation efficiency. The effects of UV light on cold $\mathrm{SiO}_{2}$ have implications for attempts to perform laser cooling on Ps confined in cryogenic cavities.

$\mathrm{Ps} X$ emission from Ge crystals is enhanced by laser irradiation, which has been previously observed in Si [42]. We have shown that this photoemission process operates at low temperatures. Low-temperature $\mathrm{Ge}$ is highly susceptible to residual gas adsorption, and Ps emission is compromised much faster than is the case for porous $\mathrm{SiO}_{2}$ samples. However, laser light that gives rise to photoemission will also desorb gas from a Ge surface, making it possible to produce Ps indefinitely in a cryogenic environment using laser irradiated $\mathrm{Ge}$.

The efficient production of low-energy Ps atoms is an important goal for Ps physics in general. Cold Ps atoms would 
be useful for many applications, including Ps Stark deceleration [14], Ps mediated antihydrogen formation [11,12], Ps-atom scattering measurements [98], precision $1^{3} \mathrm{~S}_{1} \rightarrow 2^{3} \mathrm{~S}_{1}$ optical spectroscopy [99], and microwave spectroscopy of the Ps fine structure [100-102]. The modification of existing converter materials to operate reliably at cryogenic temperatures and to produce cold Ps is a promising approach to achieving these goals. Similarly, such materials may also find application in the production of cold confined Ps, leading to studies of Ps-wall interactions [91], Ps laser cooling [22], and the formation of a Ps BEC [23].

Recently, it has been discovered that Ps can exist in delocalized Bloch states in metal-organic framework (MOF) materials [103,104], suggesting an alternative approach to producing cold Ps. Delocalized Bloch states can be emitted into vacuum with very narrow, sub-thermal energy spreads; Rydberg time-of-flight measurements [105] revealed a component of Ps with a mean energy $E_{\text {Ps }}=250 \mathrm{meV}$, with a small spread of $\sim 10 \mathrm{meV}$. MOF materials exist in a wide range of configurations [106] and can be used at cryogenic temperatures. It is not presently known what the maximum achievable Ps production efficiencies using MOF converters are, or if they are susceptible to laser induced effects such as paramagnetic center production in the same way as $\mathrm{SiO}_{2}$ films.
Owing to the unique Ps delocalization mechanism, however, they have great potential for producing monoenergetic Ps.

Further studies of silica films containing larger pores and engineered structures are warranted [34], as is an extended survey of available MOF materials. Evaluation of the efficacy of combined EPR and Ps lifetime measurements in the identification of specific paramagnetic centers could be useful in the production of Ps converters that are less sensitive to radiation at low temperatures. Moreover, owing to the surface selectivity of Ps interactions, such combined measurements could be developed as a general technique to differentiate between bulk and surface centers in EPR studies. Ps formation from various semiconductors at low temperatures, and following more rigorous surface preparation protocols, also require further study now that it has been established that Ps photoemission from $\mathrm{Ge}$ can be performed at any sample temperature.

\section{ACKNOWLEDGMENTS}

This work was supported by UCL through its Impact Studentship Programme, and was funded in part by the Leverhulme trust (Grant No. RPG-2013-055), the ERC (Grant No. CIG 630119), and the EPSRC (Grant No. EP/K028774/1).
[1] R. Paulin and G. Ambrosino, Annihilation libre de l'orthopositonium forme dans certaines poudres de grande surface specifique, J. Phys. France 29, 263 (1968).

[2] K. F. Canter, A. P. Mills, Jr., and S. Berko, Efficient Positronium Formation by Slow Positrons Incident on Solid Targets, Phys. Rev. Lett. 33, 7 (1974).

[3] A. P. Mills, Jr., Thermal activation measurement of positron binding energies at surfaces, Solid State Commun. 31, 623 (1979).

[4] P. Sferlazzo, S. Berko, K. G. Lynn, A. P. Mills, L. O. Roellig, A. J. Viescas, and R. N. West, Evidence for Phonon-assisted Positronium Emission from Graphite, Phys. Rev. Lett. 60, 538 (1988).

[5] A. P. Mills, E. D. Shaw, M. Leventhal, R. J. Chichester, and D. M. Zuckerman, Thermal desorption of cold positronium from oxygen-treated Al(111) surfaces, Phys. Rev. B 44, 5791 (1991).

[6] A. Rich, Recent experimental advances in positronium research, Rev. Mod. Phys. 53, 127 (1981).

[7] L. Liszkay, C. Corbel, P. Perez, P. Desgardin, M. F. Barthe, T. Ohdaira, R. Suzuki, P. Crivelli, U. Gendotti, A. Rubbia, M. Etienne, and A. Walcarius, Positronium reemission yield from mesostructured silica films, Appl. Phys. Lett. 92, 063114 (2008).

[8] D. W. Gidley, W. E. Frieze, T. L. Dull, A. F. Yee, E. T. Ryan, and H.-M. Ho, Positronium annihilation in mesoporous thin films, Phys. Rev. B 60, R5157(R) (1999).

[9] C. G. Fischer, M. H. Weber, C. L. Wang, S. P. McNeil, and K. G. Lynn, Positronium in low temperature mesoporous films, Phys. Rev. B 71, 180102 (2005).
[10] D. B. Cassidy, T. H. Hisakado, H. W. K. Tom, and A. P. Mills, Jr., Positronium formation via excitonlike states on $\mathrm{Si}$ and $\mathrm{Ge}$ surfaces, Phys. Rev. B 84, 195312 (2011).

[11] J. W. Humberston, M. Charlton, F. M. Jacobson, and B. I. Deutch, On antihydrogen formation in collisions of antiprotons with positronium, J. Phys. B 20, L25 (1987).

[12] A. S. Kadyrov, C. M. Rawlins, A. T. Stelbovics, I. Bray, and M. Charlton, Antihydrogen Formation Via Antiproton Scattering with Excited Positronium, Phys. Rev. Lett. 114, 183201 (2015).

[13] A. P. Mills, Jr. and M. Leventhal, Can we measure the gravitational free fall of cold Rydberg state positronium? Nucl. Instrum. Meth. 192, 102 (2002).

[14] D. B. Cassidy and S. D. Hogan, Atom control and gravity measurements using Rydberg positronium, Int. J. Mod. Phys. Conf. Ser. 30, 1460259 (2014).

[15] A. C. L. Jones, T. H. Hisakado, H. J. Goldman, H. W. K. Tom, A. P. Mills, Jr., and D. B. Cassidy, Doppler-corrected Balmer spectroscopy of Rydberg positronium, Phys. Rev. A 90, 012503 (2014).

[16] S. Chu, A. P. Mills, Jr., and J. L. Hall, Measurement of the Positronium $1^{3} S_{1}-2^{3} S_{1}$ Interval by Doppler-free Two-Photon Spectroscopy, Phys. Rev. Lett. 52, 1689 (1984).

[17] S. Mariazzi, P. Bettotti, and R. S. Brusa, Positronium Cooling and Emission in Vacuum from Nanochannels at Cryogenic Temperature, Phys. Rev. Lett. 104, 243401 (2010).

[18] D. B. Cassidy, T. H. Hisakado, H. W. K. Tom, and A. P. Mills, Jr., New Mechanism for Positronium Formation on a Silicon Surface, Phys. Rev. Lett. 106, 133401 (2011).

[19] C. H. Storry, A. Speck, D. Le Sage, N. Guise, G. Gabrielse, D. Grzonka, W. Oelert, G. Schepers, T. Sefzick, H. Pittner, 
M. Herrmann, J. Walz, T. W. Hänsch, D. Comeau, and E. A. Hessels (ATRAP Collaboration), First Laser-Controlled Antihydrogen Production, Phys. Rev. Lett. 93, 263401 (2004).

[20] A. Kellerbauer, M. Amoretti, A.S. Belov, G. Bonomi, I. Boscolo, R.S. Brusa, M. Büchner, V. M. Byakov, L. Cabaret, C. Canali, C. Carraro, F. Castelli, S. Cialdi, M. de Combarieu, D. Comparat, G. Consolati, N. Djourelov, M. Doser, G. Drobychev, A. Dupasquier, G. Ferrari, P. Forget, L. Formaro, A. Gervasini, M. G. Giammarchi, S. N. Gninenko, G. Gribakin, S. D. Hogan, M. Jacquey, V. Lagomarsino, G. Manuzio, S. Mariazzi, V. A. Matveev, J. O. Meier, F. Merkt, P. Nedelec, M. K. Oberthaler, P. Pari, M. Prevedelli, F. Quasso, A. Rotondi, D. Sillou, S. V. Stepanov, H. H. Stroke, G. Testera, G. M. Tino, G. Trnec, A. Vairo, J. Vigu, H. Walters, U. Warring, S. Zavatarelli, and D. S. Zvezhinskij, Proposed antimatter gravity measurement with an antihydrogen beam, Nucl. Instrum. Methods Phys. Res., Sect. B 266, 351 (2008).

[21] T. F. Gallagher, Rydberg Atoms (Cambridge University Press, Cambridge, 1994).

[22] E. P. Liang and C. D. Dermer, Laser cooling of positronium, Opt. Commun. 65, 419 (1988).

[23] P. M. Platzman and A. P. Mills, Jr., Possibilities for Bose condensation of positronium, Phys. Rev. B 49, 454 (1994).

[24] A. D. Cronin, J. Schmiedmayer, and D. E. Pritchard, Optics and interferometry with atoms and molecules, Rev. Mod. Phys. 81, 1051 (2009).

[25] M. Bertolotti and C. Sibilia, Coherent $\gamma$-emission by stimulated annihilation of electron-positron pairs, Appl. Phys 19, 127 (1979).

[26] R. Ramaty, F. C. Jones, and J. M. McKinley, On the theory of gamma-ray amplification through stimulated annihilation radiation, Astrophys. J. 256, 238 (1982).

[27] Y. H. Wang, B. M. Anderson, and C. W. Clark, Spinor BoseEinstein condensates of positronium, Phys. Rev. A 89, 043624 (2014).

[28] H. K. Avetissian, A. K. Avetissian, and G. F. Mkrtchian, Self-Amplified Gamma-Ray Laser on Positronium Atoms from a Bose-Einstein Condensate, Phys. Rev. Lett. 113, 023904 (2014).

[29] A. P. Mills, Jr., Possibilities with pulsed polarized high density slow positrons, J. Phys.: Conf. Ser. 505, 012039 (2014).

[30] D. B. Cassidy, V. E. Meligne, and A. P. Mills, Jr., Production of a Fully Spin-Polarized Ensemble of Positronium Atoms, Phys. Rev. Lett. 104, 173401 (2010).

[31] S. Grossmann and M. Holthaus, Bose-Einstein condensation in a cavity, Z. Phys. B: Condens. Matter 97, 319 (1995).

[32] D. B. Cassidy, T. H. Hisakado, H. W. K. Tom, and A. P. Mills, Jr., Optical Spectroscopy of Molecular Positronium, Phys. Rev. Lett. 108, 133402 (2012).

[33] A. J. Leggett, Bose-Einstein condensation in the alkali gases: Some fundamental concepts, Rev. Mod. Phys. 73, 307 (2001).

[34] C. He, S. Wang, Y. Kobayashi, T. Ohdaira, and R. Suzuki, Role of pore morphology in positronium diffusion in mesoporous silica thin films and in positronium emission from the surfaces, Phys. Rev. B 86, 075415 (2012).

[35] O. Morandi, P.-A. Hervieux, and G. Manfredi, Bose-Einstein condensation of positronium in silica pores, Phys. Rev. A 89, 033609 (2014).

[36] Y. Nagashima, M. Kakimoto, T. Hyodo, K. Fujiwara, A. Ichimura, T. Chang, J. Deng, T. Akahane, T. Chiba, K. Suzuki,
B. T. A. McKee, and A. T. Stewart, Thermalization of free positronium atoms by collisions with silica-powder grains, aerogel grains, and gas molecules, Phys. Rev. A 52, 258 (1995).

[37] C. Tianbao, X. Min, and Z. Xiangtao, Effect of the energy loss process on the annihilation of orthopositronium in silica aerogel, Phys. Lett. A 126, 189 (1987).

[38] A. P. Mills, Jr., Physics with many positrons, in Course CLXXIV "Physics with Many Positrons", Proceedings of the International School of Physics 'Enrico Fermi', edited by A. Dupasquier, R. S. Brusa, and A. P. Mills, Jr. (IOS Press, Amserdam, 2010), pp. 77-187.

[39] R. H. Dicke, The effect of collisions upon the Doppler width of spectral lines, Phys. Rev. 89, 472 (1953).

[40] H. J. Metcalf and P. van der Straten, Laser Cooling and Trapping, 1st ed. (Wiley, New York, 1999).

[41] P. Crivelli, U. Gendotti, A. Rubbia, L. Liszkay, P. Perez, and C. Corbel, Measurement of the orthopositronium confinement energy in mesoporous thin films, Phys. Rev. A 81, 052703 (2010).

[42] D. B. Cassidy, T. H. Hisakado, H. W. K. Tom, and A. P. Mills, Photoemission of Positronium from Si, Phys. Rev. Lett. 107, 033401 (2011).

[43] B. S. Cooper, A. M. Alonso, A. Deller, T. E. Wall, and D. B. Cassidy, A trap-based pulsed positron beam optimised for positronium laser spectroscopy, Rev. Sci. Instrum. 86, 103101 (2015).

[44] A. P. Mills, Jr. and E. M. Gullikson, Solid neon moderator for producing slow positrons, Appl. Phys. Lett. 49, 1121 (1986).

[45] R. G. Greaves and J. Moxom, Design and performance of a trap based positron beam source, AIP Conf. Proc. 692, 140 (2003).

[46] C. M. Surko, M. Leventhal, and A. Passner, Positron Plasma in the Laboratory, Phys. Rev. Lett. 62, 901 (1989).

[47] J. R. Danielson, D. H. E. Dubin, R. G. Greaves, and C. M. Surko, Plasma and trap-based techniques for science with positrons, Rev. Mod. Phys. 87, 247 (2015).

[48] A.P. Mills, Jr., Time bunching of slow positrons for annihilation lifetime and pulsed laser photon absorption experiments, Appl. Phys. 22, 273 (1980).

[49] D. B. Cassidy, P. Crivelli, T. H. Hisakado, L. Liszkay, V. E. Meligne, P. Perez, H. W. K. Tom, and A. P. Mills, Jr., Positronium cooling in porous silica measured via Doppler spectroscopy, Phys. Rev. A 81, 012715 (2010).

[50] A. Deller, B. S. Cooper, T. E. Wall, and D. B. Cassidy, Positronium emission from mesoporous silica studied by laserenhanced time-of-flight spectroscopy, New J. Phys. 17, 043059 (2015).

[51] D. B. Cassidy, S. H. M. Deng, H. K. M. Tanaka, and A. P. Mills, Jr., Single shot positron annihilation lifetime spectroscopy, Appl. Phys. Lett. 88, 194105 (2006).

[52] D. B. Cassidy and A. P. Mills, Jr., A fast detector for singleshot positron annihilation lifetime spectroscopy, Nucl. Instrum. Meth. A 580, 1338 (2007).

[53] L. L. Hench and J. K. West, The sol-gel process, Chem. Rev. 90, 33 (1990).

[54] W. Yantasee, Y. Lin, X. Li, G. E. Fryxell, T. S. Zemanian, and V. V. Viswanathan, Nanoengineered electrochemical sensor based on mesoporous silica thin-film functionalized with thiolterminated monolayer, Analyst 128, 899 (2003).

[55] S. J. Tao, Positronium in molecular substances, Appl. Phys. 3, 1 (1974). 
[56] W. Brandt and R. Paulin, Positronium Diffusion in Solids, Phys. Rev. Lett. 21, 193 (1968).

[57] Y. Nagashima, Y. Morinaka, T. Kurihara, Y. Nagai, T. Hyodo, T. Shidara, and K. Nakahara, Origins of positronium emitted from $\mathrm{SiO}_{2}$, Phys. Rev. B 58, 12676 (1998).

[58] P. Sferlazzo, S. Berko, and K. F. Canter, Time-of-flight spectroscopy of positronium emission from quartz and magnesium oxide, Phys. Rev. B 35, 5315 (1987).

[59] A. P. Mills, Jr., E. D. Shaw, R. J. Chichester, and D. M. Zuckerman, Production of slow positron bunches using a microtron accelerator, Rev. Sci. Instrum. 60, 825 (1989).

[60] C. J. Brinker, Y. Lu, A. Sellinger, and H. Fan, Evaporationinduced self-assembly: Nanostructures made easy, Adv. Mater. 11, 579 (1999).

[61] D. Grosso, F. Cagnol, G. J. de A. A. Soler-Illia, E. L. Crepaldi, H. Amenitsch, A. Brunet-Bruneau, A. Bourgeois, and C. Sanchez, Fundamentals of mesostructuring through evaporation-induced self-assembly, Adv. Funct. Mater. 14, 309 (2004).

[62] L. Liszkay, M.-F. Barthe, C. Corbel, P. Crivelli, P. Desgardin, M. Etienne, T. Ohdaira, P. Perez, R. Suzuki, V. Valtchev, and A. Walcarius, Orthopositronium annihilation and emission in mesostructured thin silica and silicalite-1 films, Appl. Surf. Sci. 255, 187 (2008).

[63] A. P. Mills, Jr., E. D. Shaw, R. J. Chichester, and D. M. Zuckerman, Positronium thermalization in $\mathrm{SiO}_{2}$ powder, Phys. Rev. B 40, 2045 (1989).

[64] D. B. Cassidy and A. P. Mills, Jr., Enhanced Ps-Ps Interactions Due to Quantum Confinement, Phys. Rev. Lett. 107, 213401 (2011).

[65] M. Imperor-Clerc, P. Davidson, and A. Davidson, Existence of a microporous corona around the mesopores of silica-based SBA-15 materials templated by triblock copolymers, J. Am. Chem. Soc. 122, 11925 (2000).

[66] L. Liszkay, F. Guillemot, C. Corbel, J.-P. Boilot, T. Gacoin, E. Barthel, P. Pérez, M.-F. Barthe, P. Desgardin, P. Crivelli, U. Gendotti, and A. Rubbia, Positron annihilation in latextemplated macroporous silica films: pore size and orthopositronium escape, New J. Phys. 14, 065009 (2012).

[67] S. Mariazzi, P. Bettotti, S. Larcheri, L. Toniutti, and R. S. Brusa, High positronium yield and emission into the vacuum from oxidized tunable nanochannels in silicon, Phys. Rev. B 81, 235418 (2010)

[68] G. W. Ford, L. M. Sander, and T. A. Witten, Lifetime Effects of Positronium in Powders, Phys. Rev. Lett. 36, 1269 (1976).

[69] C. He, T. Ohdaira, N. Oshima, M. Muramatsu, A. Kinomura, R. Suzuki, T. Oka, and Y. Kobayashi, Evidence for pore surface dependent positronium thermalization in mesoporous silica/hybrid silica films, Phys. Rev. B 75, 195404 (2007).

[70] S. Mariazzi, L. Toniutti, N. Patel, and R. S. Brusa, Formation and escaping of positronium in porous $\mathrm{SiO}_{2}$ films at low temperature, Appl. Surf. Sci. 255, 191 (2008)

[71] F. Moia, R. Ferragut, A. Dupasquier, M. G. Giammarchi, and G. Q. Ding, Thermal production of positronium in porous alumina, Eur. Phys. J. D 66, 124 (2012).

[72] D. W. Gidley, H. G. Peng, and R. S. Vallery, Positron annihilation as a method to characterize porous materials, Annu. Rev. Mater. Res. 36, 49 (2006).
[73] R. Krause-Rehberg and H. S. Leipner, Positron Annihilation in Semiconductors: Defect Studies, Springer Series in Solid-State Sciences (Springer, Berlin, Heidelberg, 2010).

[74] M. Deutsch, Evidence for the formation of positronium in gases, Phys. Rev. 82, 455 (1951).

[75] Richard A. Ferrell, Ortho-parapositronium quenching by paramagnetic molecules and ions, Phys. Rev. 110, 1355 (1958).

[76] H. Saito and T. Hyodo, Quenching of positronium by surface paramagnetic centers in ultraviolet- and positron-irradiated fine oxide grains, Phys. Rev. B 60, 11070 (1999).

[77] I. A. Shkrob and A. D. Trifunac, Time-resolved epr of spinpolarized mobile $h$ atoms in amorphous silica: The involvement of small polarons, Phys. Rev. B 54, 15073 (1996).

[78] C. Dauwe and Mbungu-Tsumbu, Orthopositronium study of positron-irradiation-induced surface defects in alumina powder, Phys. Rev. B 45, 9 (1992).

[79] A. F. Lazzarini and E. Lazzarini, Metal electron delocalization in $3 \mathrm{~d}$ complexes estimated by positronium spin-exchange reactions, Coord. Chem. Rev. 213, 159 (2001).

[80] H. Saito, Y. Nagashima, T. Hyodo, and T. Chang, Detection of paramagnetic centers on amorphous- $\mathrm{SiO}_{2}$ grain surfaces using positronium, Phys. Rev. B 52, R689(R) (1995).

[81] H. J. Zhang, Z. Q. Chen, S. J. Wang, A. Kawasuso, and N. Morishita, Spin conversion of positronium in $\mathrm{NiO} / \mathrm{Al}_{2}$ $\mathrm{O}_{3}$ catalysts observed by coincidence Doppler broadening technique, Phys. Rev. B 82, 035439 (2010).

[82] C. Dauwe, B. Van Waeyenberge, D. Segers, T. Van Hoecke, and J. Kuriplach, Some aspects of the interactions of orthopositronium with perfect and defect surfaces of insulating materials, J. Radioanal. Nucl. Chem. 210, 293 (1996).

[83] N. Djourelov, T. Suzuki, V. Shantarovich, and K. Kondo, Positronium formation in sol-gel-prepared silica-based glasses: temperature and positron-irradiation effect, Radiation Phys. Chem. 72, 723 (2005).

[84] D. B. Cassidy and A. P. Mills, Jr., Radiation damage in $a-\mathrm{SiO}_{2}$ exposed to intense positron pulses, Nucl. Instrum. Methods Phys. Res., Sect. B 262, 59 (2007).

[85] D. B. Cassidy and A. P. Mills, Interactions between Positronium Atoms in Porous Silica, Phys. Rev. Lett. 100, 013401 (2008).

[86] D. B. Cassidy, K. T. Yokoyama, S. H. M. Deng, D. L. Griscom, H. Miyadera, H. W. K. Tom, C. M. Varma, and A. P. Mills, Positronium as a probe of transient paramagnetic centers in $a-\mathrm{SiO}_{2}$, Phys. Rev. B 75, 085415 (2007).

[87] P. J. Schultz and K. G. Lynn, Interaction of positron beams with surfaces, thin films, and interfaces, Rev. Mod. Phys. 60, 701 (1988).

[88] A. P. Mills, Jr. and R. J. Wilson, Transmission of 1-6 keV positrons through thin metal films, Phys. Rev. A 26, 490 (1982).

[89] J. Algers, P. Sperr, W. Egger, G. Kögel, and F. H. J. Maurer, Median implantation depth and implantation profile of 3-18 keV positrons in amorphous polymers, Phys. Rev. B 67, 125404 (2003).

[90] S. L. Andersen, D. B. Cassidy, J. Chevallier, B. S. Cooper, A. Deller, T. E. Wall, and U. I. Uggerhøj, Positronium emission and cooling in reflection and transmission from thin mesostructured silica films, J. Phys. B 48, 204003 (2015).

[91] D. B. Cassidy, M. W. J. Bromley, L. C. Cota, T. H. Hisakado, H. W. K. Tom, and A. P. Mills, Jr., Cavity Induced Shift and 
Narrowing of the Positronium Lyman- $\alpha$ Transition, Phys. Rev. Lett. 106, 023401 (2011).

[92] M. Weinelt, M. Kutschera, T. Fauster, and M. Rohlfing, Dynamics of Exciton Formation at the $\mathrm{Si}(100) \mathrm{c}(4 \times 2)$ Surface, Phys. Rev. Lett. 92, 126801 (2004).

[93] T. Ichibayashi and K. Tanimura, Ultrafast Carrier Relaxation in Si Studied by Time-Resolved Two-Photon Photoemission Spectroscopy: Intravalley Scattering and Energy Relaxation of Hot Electrons, Phys. Rev. Lett. 102, 087403 (2009).

[94] T. Sjodin, H. Petek, and H. L. Dai, Ultrafast Carrier Dynamics in Silicon: A Two-color Transient Reflection Grating Study on a (111) Surface, Phys. Rev. Lett. 81, 5664 (1998).

[95] A. Deller, D. Edwards, T. Mortensen, C. A. Isaac, D. P. van der Werf, H. H. Telle, and M. Charlton, Exciting positronium with a solid-state UV laser: The Doppler-broadened Lyman- $\alpha$ transition, J. Phys. B 48, 175001 (2015).

[96] K. P. Ziock, C. D. Dermer, R. H. Howell, F. Magnotta, and K. M. Jones, Optical saturation of the $1^{3} \mathrm{~S}-2^{3} \mathrm{P}$ transition in positronium, J. Phys. B 23, 329 (1990).

[97] T. E. Wall, A. M. Alonso, B. S. Cooper, A. Deller, S. D. Hogan, and D. B. Cassidy, Selective Production of Rydberg-Stark States of Positronium, Phys. Rev. Lett. 114, 173001 (2015).

[98] S. J. Brawley, S. E. Fayer, M. Shipman, and G. Laricchia, Positronium Production and Scattering Below its Breakup Threshold, Phys. Rev. Lett. 115, 223201 (2015).

[99] M. S. Fee, A. P. Mills, Jr., S. Chu, E. D. Shaw, K. Danzmann, R. J. Chichester, and D. M. Zuckerman, Measurement of the
Positronium $1^{3} \mathrm{~S}_{1}-2^{3} \mathrm{~S}_{1}$ Interval by Continuous-Wave TwoPhoton Excitation, Phys. Rev. Lett. 70, 1397 (1993).

[100] A. P. Mills, Jr., S. Berko, and K. F. Canter, Fine-Structure Measurement in the First Excited State of Positronium, Phys. Rev. Lett. 34, 1541 (1975).

[101] S. Hatamian, R. S. Conti, and A. Rich, Measurements of the $2^{3}$ $\mathrm{S}_{1}-2^{3} \mathrm{P}_{j}(j=0,1,2)$ Fine-Structure Splittings in Positronium, Phys. Rev. Lett. 58, 1833 (1987).

[102] D. Hagena, R. Ley, D. Weil, G. Werth, W. Arnold, and H. Schneider, Precise Measurement of $n=2$ Positronium FineStructure Intervals, Phys. Rev. Lett. 71, 2887 (1993).

[103] D. Dutta, J. I. Feldblyum, D. W. Gidley, J. Imirzian, M. Liu, A. J. Matzger, R. S. Vallery, and A. G. Wong-Foy, Evidence of Positronium Bloch States in Porous Crystals of $\mathrm{Zn}_{4} \mathrm{O}$-Coordination Polymers, Phys. Rev. Lett. 110, 197403 (2013).

[104] P. Crivelli, D. Cooke, B. Barbiellini, B. L. Brown, J. I. Feldblyum, P. Guo, D. W. Gidley, L. Gerchow, and A. J. Matzger, Positronium emission spectra from self-assembled metal-organic frameworks, Phys. Rev. B 89, 241103 (2014).

[105] A. C. L. Jones, H. J. Goldman, Q. Zhai, P. Feng, H. W. K. Tom, and A. P. Mills, Jr., Monoenergetic Positronium Emission from Metal-Organic Framework Crystals, Phys. Rev. Lett. 114, 153201 (2015).

[106] C. Janiak and J. K. Vieth, MOFs, MILs and more: Concepts, properties and applications for porous coordination networks (PCNs), New J. Chem. 34, 2366 (2010). 